\title{
Assimilation and processing of observation data obtained by satellite earth sensing for monitoring the current state of heterogeneous objects on the water surface
}

\author{
Natalia Panasenko ${ }^{1,}$, , Nikolay Motuz ${ }^{1}$, Asya Atayan ${ }^{1}$ \\ ${ }^{1}$ Don State Technical University, 1, Gagarin sq., Rostov-on-Don, 344003, Russia
}

\begin{abstract}
The study is devoted to the analysis of satellite observations data assimilation to discover the necessary information for developing and verifying mathematical models of hydrodynamics and biological shallowwater kinetics. The use of satellite earth sensing data is taken to enhance information base. The possible use of neural networks with optical flow computation is considered in the study. The objective of the study is to develop a software tool used to identify the initial conditions in mathematical modeling of hydrobilogical shallow-water processes.
\end{abstract}

\section{Introduction}

In the past decade, the frequency of the happened adverse and disastrous phenomena in the coastal South Russian systems has increased. The list of events contains the following disasters: the calamitous storm in November 2006; the storm surges in 2007 and 2014, which caused loss of life and destruction; the shallowing of the Azov Sea nearby Taganrog (Rostov region) and the river Don in November 2019, which related to the poor rainfall in the river basins flow into the Azov Sea and the strong wind made the coast grow shallow promptly; the massive fish kill in July 2020 in the south-eastern sector of the Azov Sea, which greatly damaged commercial fish resources. The list is not complete with such phenomena and processes which are necessary to be predicted using interrelated models of hydrodynamics and biological kinetics.

A huge number of Russian and foreign scientists are engaged in mathematical modeling of hydrodynamics process and biological kinetics of nature system problems, which are represented by coastal and marine systems. Fundamental scientific works of such authors as Lotka A.J. [1], Volterra V. [2], Marchuk G.I. [3], Ozmidov R. V. [4], Gause G.F. [5] greatly contributed to field of creating mathematical models, developing methods for diagnosing and predicting changes in aquatic ecosystems, these works considered the problem of interspecific interaction. Hutchinson G. E. studied the flora and fauna of lakes. Mitscherlich E. A. discovered the law of action of growth factors and presented a new view on the science of plants [6]. Vinberg G.G. [7] was engaged in research related to the processes of nutrition

\footnotetext{
*Corresponding author: natalija93_93@mail.ru
} 
and growth of aquatic organisms. Vorovich I.I., and Gorstko A.B. [8] developed schemes for the rational use of water resources. Odum H.T. explained ecological structures from energy systems [9]. Abakumov A.I. [10] is actively engaged in the development of methods for optimizing the modeling of aquatic ecosystems. Jorgensen S.E., Meyer H., Firiis M. [11] studied the issues of Limnology and ecological modeling. Vollenweider R.A. investigated the eutrophication of lakes. Berdnikova S.V. [12], Ilyina G.V. [13], Ilyicheva V.G. [14] examined mathematical modeling of the contaminants spreading in coastal systems. Despite the great amount of models all they serve the purpose to the single gain - creating an accurate description of aquatic ecosystems functioning.

Nowadays the problem of equipping these systems with real input database for their analysis and forecasting is a burning issue, it can make possible to set the correct initialboundary conditions for the systems of non-linear equation with a partial derivative and determine the coefficients of these factors and other functional dependencies which are included to the constructed models. The scientific and technological progress develops new methods of ocean research due to the global Earth changes studies (studies by Marchuk G.I. $[15,16,17]$, Shutyaev V.P. [18, 19, 16] and Agoshkov V.I. [19, 17, 20]).

There is increasing interest to the problem of assimilation and processing of observation data obtained by high accurate satellite earth sensing (SES) which allow monitor the current state of oceans.

Data obtained by satellite earth sensing make possible not only to equip mathematical models with the necessary information (boundary, initial conditions, information about source functions) but also to assimilate the constructed output sensing data with a view to improving the accuracy and increasing the reliability of predictive modeling. The most important task is to be solved consists in development of methods and tools for the heterogeneous satellite data applying. In this regard, the purpose of this work is to describe assimilation and processing of observation data obtained by satellite earth sensing for monitoring the current state of heterogeneous objects on the water surface, in particular, the processes of "blooming" of phytoplankton algae.

\section{Problem statement}

In the last decade, satellite earth sensing methods have become essential for studying environmental and land management, researching plant communities, assessing agricultural crops and the impact of natural disasters. Nowadays there have been launched a great amount of satellites (among them are Resurs-P, WorldView, Landsat, KompSat, SPOT, Kanopus-V, GeoEye, Radarsat, Resurs-F and many others), both Russian and foreign satellites cover most of the Earth. Despite this there is a serious problem to get "cloudless" snapshots and snapshots at various scales. Going on the further studying of monitoring the current state of water surface, in particular the phytoplankton "blooming" process, define the following notions:

Phytoplankton is the base of the trophic pyramid of the shallow water reservoir biogeocenosis, the main link in the food chain «phytoplankton-zooplanktonfish». With the flow of rivers into reservoirs come nutrients-compounds of nitrogen, silicon, and phosphorus, causing a surge in the growth of the phytoplankton population, called «blooming» [21].

The problem of intensive water surface "blooming" is very acute for a wide variety of aquatic areas. Water "blooming" is called the rapid growth of one or 2-3 planktonic species, which causes water quality deterioration. Intensive and often detrimental phytoplankton "blooming" of both fresh and marine water has increased dramatically over the past 20-30 years. Hydrobiologists explain the reason by a whole range of factors, such as climate 
changing, entering a large amount of various mineral and organic substances into the water, which associated with the human economic activity intensification [23].

The vortex flows of various spatial scale has a significant meaning for aquatic areas similar to the Azov Sea and other south Russian coastal systems. The expeditionary measurements analysis results of the Azov Sea water-related indicators described in [24] and satellite earth sensing data taken the snapshots of the visible range point out kill fish phenomena process in the Central-Eastern region, which happens seasonally. The areas of intensive "blooming" and structures of vortex flows look well-defined at the satellite earth sensing snapshots. The large-scale marks of a cyclonic vortex realize an example of how biogenic pollutants caused by an intense cyanobacteria blooming spread under the current dynamic and circulating processes. Fig. 1 represents the color-synthesized snapshots from the WorldView [26] and Resurs-P [25] satellites, gotten on September 10, 2019 over the Azov Sea aquatic area.

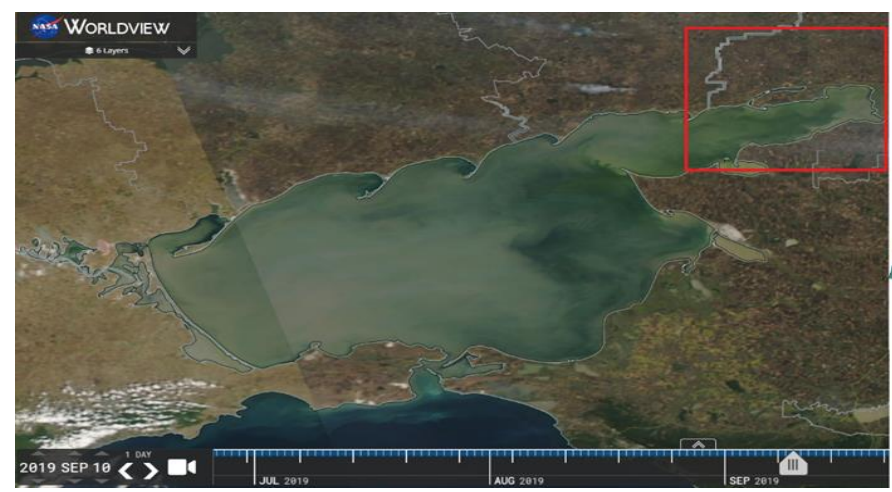

a)

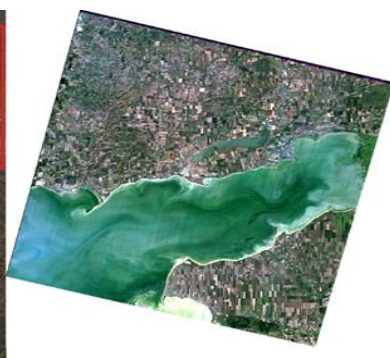

b)

Fig. 1. Propagation of impurities: a) WorldView satellite; b) Resource-P satellite.

\subsection{Mathematical application}

Consider a mathematical model of phytoplankton evolution. Figure 2 shows a diagram of a water body in the form of a closed pool, bounded: $\sigma$ by side surface of a water body; $\Sigma 0$ by surface of the sea; $\Sigma H=(x, y)$ by the bottom surface. $\Sigma=\Sigma_{0} \cup \Sigma_{H} \cup \sigma, \Sigma$ is the domain boundary $G$, which is piecewise smooth for all $t \in\left(0, T_{0}\right][21,22]$.

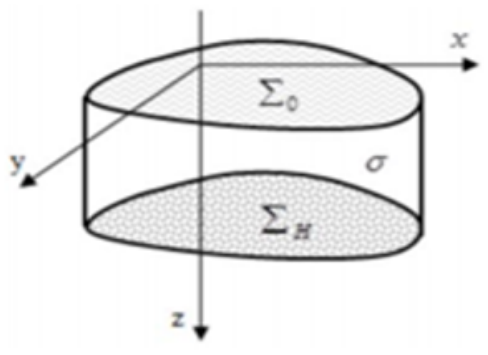

Fig. 2. Water-body scheme.

This task needs to consider a great number of parameters, some of which can be observed using high precision measurement from satellite earth sensing. These parameters include the initial phytoplankton distribution, the water temperature and many others. 


\section{Neural network}

Artificial neural networks are considered to be a rather breakthrough technology, though they were descripted at the level of algorithms in the second half of the last century. But humanity has accumulated a sufficient amount of information and created the technical capabilities to implement them into final products only at the present time.

Neural networks can be used for many tasks, including data analysis, optimization, and compression, approximation, prediction, clustering, decision making, and detection. Each of these directions can be used in solving hydrodynamics and biological kinetics model problems, but mostly neural networks are used for detection - recognition, and classification of images [21].

Our goal is to teach the neural network to construct the movements of different objects, which may be marks of phytoplankton population or surface covers including oil pollution, using adjacent snapshots (close in shooting time). It is necessary to develop software with the ability of computing the optical flow between snapshots using supervised neural network training.

Creating the input data for processing needs to collect snapshots, for example, consider the color-synthesized satellite imagery from WorldView (having rather blurred boundaries) [26], obtained on September 10, 2019 over the Azov Sea at different times of the day. These snapshots are splitted up into smaller fragments and called training data (figure 3 ).

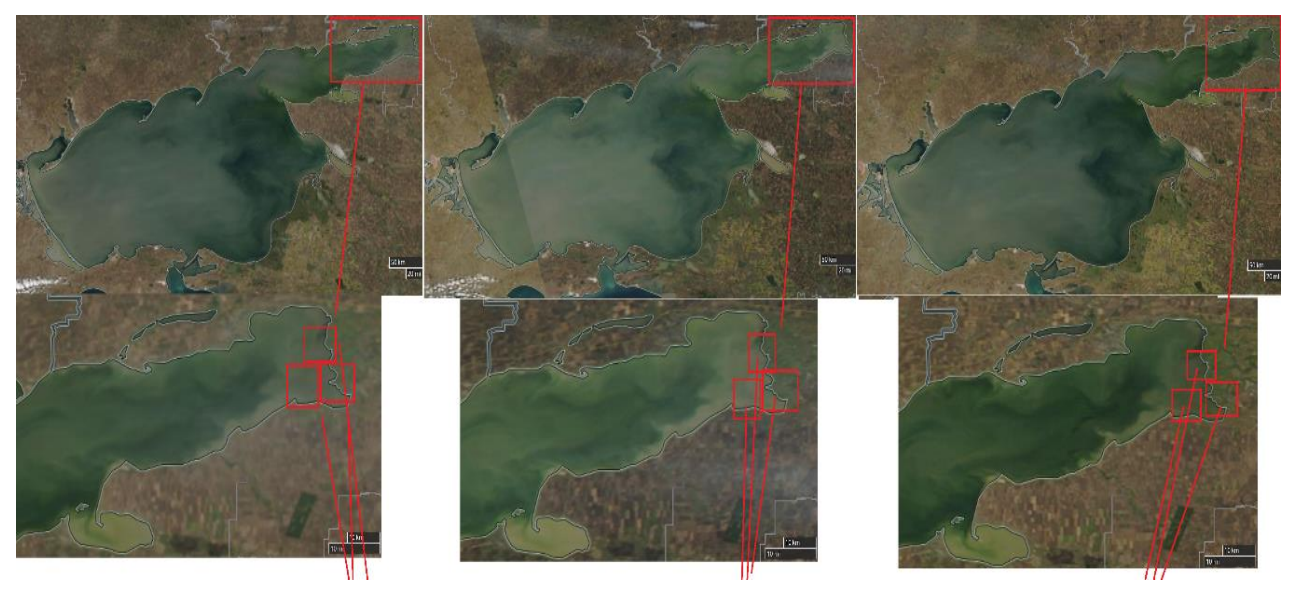

Fig. 3. Propagation of impurities WorldView satellite.

The basic idea of this technology is to improve the accuracy of predictive modeling. A wide collection of snapshots is necessary for the successful implementation. The software is implemented in Python. This will allow us to identify and classify the areas of phytoplankton distribution and pollutant impurities.

\section{Discussion and conclusions}

The article illustrates the relevance of applying the observational data processing from satellite earth sensing. There are considered the necessity and possibility of using the neural network approach with the optical flow computation in solving problems of information processing from gray-scale snapshots. We will rely the further research on the obtained results. The software customization is planned for the computer implementation of processing. We will consider the mathematical side of this issue more thoroughly. 


\section{Acknowledgements}

The reported study was funded by RFBR, project number 19-37-90070. The reported study was funded by RFBR, project number 20-31-90105.

\section{References}

1. Lotka A J 1922 Proc Natl Acad Sci 8 147-150 DOI:101073/pnas86147

2. Volterra V 1928 Rapp P - V Reun Cons Int Explor Mer 3 3-51 DOI:101093/icesjms/313

3. Marchuk G I, Sarkisyan A S 1988 Mathematical modeling of ocean circulation (Moscow: Nauka) p 304

4. Ozmidov R V 1986 Diffusion of impurities in the ocean (L: Hydrometeoizdat) p 278

5. Gause G F 1932 Journal of Experimental Biology 9 389-402

6. Mitscherlich E A 1909 Das Gesert des Minimums und das Gesetz des abnehmenden Bodenertrags (Landw Jahrb) p 595

7. Vinberg G G 1985 Production of populations and communities of aquatic organisms and methods of its study Sverdlovsk (Ural center of the USSR Academy of Sciences) $\mathrm{p}$ 13-18

8. Vorovich I I, Gorelov A S, Gorstko A B, Dombrovsky Yu A, Zhdanov Yu A, Surkov F A, Epstein L V 1981 Rational use of water resources of the Azov sea basin: mathematical models (Moscow: Nauka) p 360

9. Odum H T 1983 System Ecology (New York: Wiley) p 644

10. Abakumov A I 2010 Proceedings Of the Institute of system analysis of the Russian Academy of Sciences System analysis of the problem of sustainable development 54 4960

11. Jorgensen SE, Mejer H, Firiis M 1978 Ecological Modelling 4 253-278

12. Berdnikov S V 1989 Marine Hydrophysical Journal 3 52-58

13. Ilyichev V G 2008 Sustainability, adaptation and management in ecological systems (M: Publishing house FIZMATLIT) p 231

14. Isaeva L S 1963 Proceedings of MGI Physics of the sea (K: Publishing house of the Academy of Sciences of the Ukrainian SSR) 28 36-39

15. Marchuk G I, Paton B E, Korotaev G K, Zalesny V B 2013 Izvestiya Atmospheric and Oceanic Physics 49(6) 579-591

16. Marchuk G I, Shutyaev V P 2011 Proceedings of the Institute of Mathematics and Mechanics, Ural Branch of the Russian Academy of Sciences 17(2) 136-150

17. Marchuk G I, Agoshkov V I, Ipatova V M 2011 Proceedings of the Moscow Institute of Physics and Technology 3.1(9) 93-101

18. Shutyaev V P 2019 Izvestiya Atmospheric and Oceanic Physics 55(1) 17-31

19. Agoshkov V I, Parmuzin E I, Zakharova N B, Shutyaev V P 2018 Russian Journal of Numerical Analysis and Mathematical Modelling 33(3) 149-160

20. Agoshkov V I, Zalesny V B, Sheloput T O 2020 Izvestia of the Russian Academy of Sciences Physics of the atmosphere and ocean 56(3) 293-308

21. Leontyev A L, Nikitina A V, Chumak M I 2020 Computational Mathematics and Information Technologies Electronic journal 1(1) 1-11

22. Nikitina A V, Sukhinov A I, Peskova O Yu 2001 Izvestia TRTU 2(20) 32-35 
23. Lavrova O Yu, Soloviev D M, Strochkov A Ya, Shendrik V D 2014 Sovremennye problemy distantsionnogo zondirovaniya Zemli iz kosmosa 11(3) 54-72

24. Sukhinov A I, Atayan A M, Belova Yu V, Litvinov V N, Nikitina A V, Chistyakov A E 2020 Computational Continuum Mechanics 13(2) 161-174

25. Roscosmos Geoportal https://wwwgptlru

26. NASA Worldview http://worldviewearthdatanasagov

27. Rostovtsev V S 2019 Artificial neural networks (St Petersburg: Lan) p 216 